\title{
Patient Specific Models for Planning and Guidance of Minimally Invasive Aortic Valve Implantation
}

\author{
I. Waechter ${ }^{1}$, R. Kneser ${ }^{1}$, G. Korosoglou ${ }^{2}$, J. Peters ${ }^{1}$, \\ N.H. Bakker ${ }^{3}$, R.v.d. Boomen ${ }^{3}$, and J. Weese ${ }^{1}$ \\ 1 Philips Research Aachen, Germany \\ 2 Universitaet Heidelberg, Kardiologie, Germany \\ 3 Interventional X-Ray, Philips Healthcare, Best, The Netherlands
}

\begin{abstract}
Recently, new techniques for minimally invasive aortic valve implantation have been developed generating a need for planning tools that assess valve anatomy and guidance tools that support implantation under x-ray guidance. Extracting the aortic valve anatomy from CT images is essential for such tools and we present a model-based method for that purpose. In addition, we present a new method for the detection of the coronary ostia that exploits the model-based segmentation and show, how a number of clinical measurements such as diameters and the distances between aortic valve plane and coronary ostia can be derived that are important for procedure planning. Validation results are based on accurate reference annotations of $20 \mathrm{CT}$ images from different patients and leave-one-out tests. They show that model adaptation can be done with a mean surface-to-surface error of $0.5 \mathrm{~mm}$. For coronary ostia detection a success rate of $97.5 \%$ is achieved. Depending on the measured quantity, the segmentation translates into a root-mean-square error between $0.4-1.2 \mathrm{~mm}$ when comparing clinical measurements derived from automatic segmentation and from reference annotations.
\end{abstract}

\section{Introduction}

Valvular heart diseases are among the most prominent causes of heart failure and premature cardiac death. Aortic valve stenosis is a very common valvular disease and aortic valve replacement in open surgery has been conducted on these patients for decades. This is, however, a very invasive and expensive treatment. In addition, it is considered too high risk or contraindicated for many patients [1]. In the last decade, techniques for minimally invasive aortic valve implantation have been developed [1/2] that offer a new treatment option. The artificial valve is mounted on a stent which is delivered through a catheter, either transfemoral, transsubclavian, or transapical, under X-ray guidance. Accurate assessment of the valve anatomy is essential as, e.g. occlusion of the coronary ostia by the valve leaflets or the rim of the stent is life threatening. Furthermore, methods to guide implantation are important as the aortic valve anatomy is not clearly visible when using X-ray imaging. 
In this paper, we present a method to extract the aortic valve anatomy from CT images. The method is based on a model-based segmentation approach previously developed to segment the heart from CT images 3 . In addition, a new method for the detection of anatomical landmarks is presented that exploits the model-based segmentation. Within the reference model, the likely regions of the coronary ostia have been encoded to support their automatic detection. The aortic valve model refers to a heart phase with closed aortic valve as overpacing is used to stop the heart during implantation. In addition, it covers not only the aortic valve and bulbus, but also the aorta and the left ventricle, which are of relevance when using the transfemoral or transapical approach, respectively. The model differs in that respect from the work of Ionasec et al. 4] who have presented methods to generate a dynamic model of the aortic valve only.

Furthermore, we show how a number of measurements that are important for stent selection and procedure planning (see [5] for relevant measurements) can be derived from the model and information encoded therein . Examples are the distance of the coronary ostia to the valve plane and diameter measurements along the outflow tract, the aortic bulbus, and the ascending aorta. Extraction of the measurements is done automatically, which distinguishes the work from Gessat et al. 6] who presented a planning system for transapical valve implantation relying on user selected anatomical landmarks.

The paper is structured as follows: In section 2, we present our approach for the segmentation of the aortic valve and the new anatomical landmark detection method that we use to localize the coronary ostia. In addition, we describe how we determine the planes for measurements and how we compute the desired measurements. Section 3 includes validation results for the segmentation accuracy, landmark detection, and measurement accuracy.

\section{Method}

\subsection{Model-Based segmentation}

Previously, a method for segmenting the heart chambers and the great vessels [37] was presented. The method detects the heart with an adapted Generalized Hough Transform and positions a generic heart model in the image. Mesh adaptation is done by iterating boundary detection and mesh deformation.

The pose of the heart chambers is improved by minimizing the external energy $E_{\text {ext }}$ with respect to the parameters of a global similarity transformation during mesh deformation. After 20 iterations, the point of gravity of the most distal descending aorta segment is computed, and the descending aorta is detected in the corresponding axial image slice. Another 20 iterations are performed to refine adaptation of the heart chambers and the most distal descending aorta segment, where a linear transformation is assigned to each model part and the external energy $E_{\text {ext }}$ is minimized with respect to the parameters of the multi-linear transformation. Finally, 30 iterations of deformable adaptation are performed. The tubular segments building the great vessels are successively activated during these iterations. Mesh deformation is done by adding shape constraints to 
the energy $E=E_{\text {ext }}+\alpha E_{\text {int }}$. In particular, the internal energy penalizes deviations from the generic heart model undergoing a multi-linear transformation to account for pose and shape variability.

For acceleration, mesh adaptation is largely performed with a reduced mesh resolution. In addition, already well-adapted mesh parts are frozen during deformable adaptation. Robustness is achieved by assigning optimal boundary detection functions with Simulated Search [8].

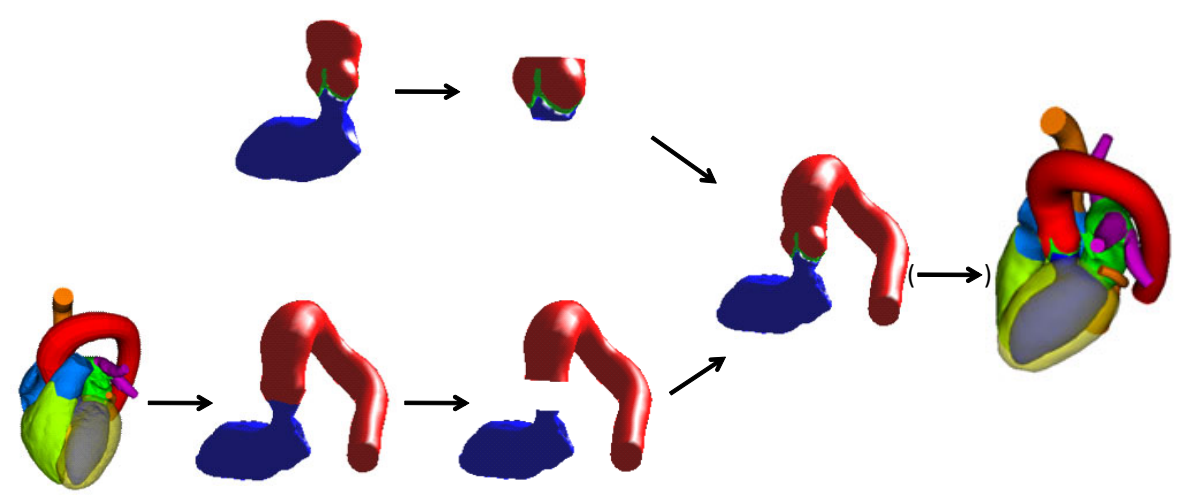

Fig. 1. Merging two models: The upper model contains the detailed aortic valve and aortic bulbus. These structures are cut and inserted into the lower, whole heart model.

Within the existing heart model, the aortic valve and aortic bulbus have only roughly been modelled. Therefore, a more detailed model of the aortic valve, aortic bulbus, and left ventricle was created. For that purpose, these structures were manually annotated (and reviewed by a clinician) in $20 \mathrm{CT}$ datasets resulting in 20 reference meshes. These were used to build a mean mesh model and to train boundary detection functions by simulated search [8. The aortic valve model was subsequently integrated into the heart model (see Fig. 1). For that purpose, both models were adapted to the same patient to bring them in the same coordinate system. Both meshes were cut with identical cut planes, one below the aortic valve and one above the aortic bulbus. Finally, the aortic valve model was attached to the left ventricle and the aorta of the heart model. The other parts of the heart model can be added if required by the application. All mesh-related information, like anatomical affiliations and boundary detectors, were transferred from one of the models to the merged model.

\subsection{Ostia Finder}

The shape, diameter, appearance, and position of the coronary ostia vary substantially between patients (see Fig. 2). This makes it difficult to detect them: including the ostia directly in the model is difficult as their position varies too much; a landmark detection method is also difficult as similar structures in the dataset may lead to many false detection results. We propose, therefore, a new approach. The basic idea is to use information from model-based segmentation 

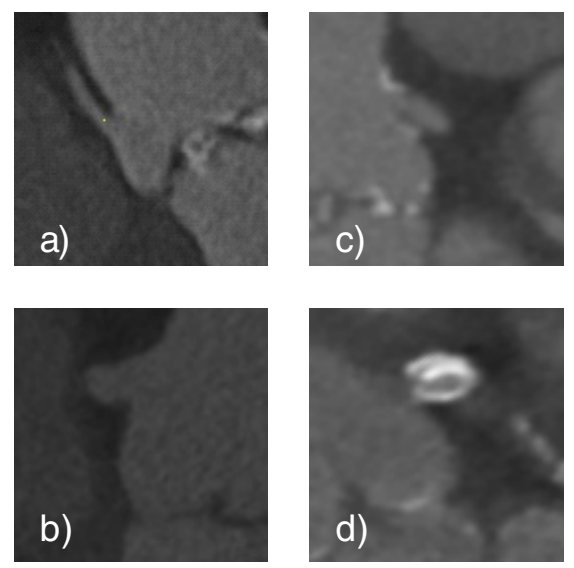

Fig. 2. Examples of Ostia: a) thin and pointing straight up b) thick and curving downwards c) pointing straight down, very near to aortic bulbus d) poor visibility of first part then calcified

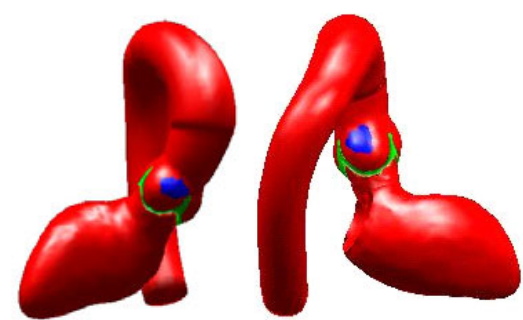

Fig. 3. Ostia candidate patches

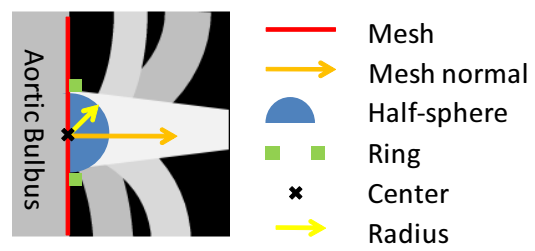

Fig. 4. Ostia search pattern with potential shapes of ostia

and to restrict landmark detection for the coronary ostia to a well-defined area on the surface of the aortic bulbus.

To encode the area for coronary ostia detection in the model, the mesh vertex closest to a coronary ostium is determined and the triangles linked to the vertex are marked. This is done for all manually determined coronary ostia positions in the training images. Fig. 3 shows the resulting candidate patches of both coronary ostia. They have a diameter between $1 \mathrm{~cm}$ and $1.5 \mathrm{~cm}$.

Coronary ostia detection itself presumes that model-based segmentation has been performed. The candidate patch is determined for the actual image and and pattern matching is performed on the surface of the patch. The pattern consist of a half-sphere $S(c, r)$ and a ring $R(c, r)$ (see Fig. 4), where $r$ is the radius of the half-sphere and the inner radius of the ring and $c$ is the centre of both. The orientation of the half-sphere and the ring is given by the mesh normal at the centre $c$. Assuming that the correct ostium position is characterized by a high image intensity inside the ostium that is surrounded by a ring with low image intensity, the likelihood for a coronary ostium is computed according to

$$
L(c, r)=\frac{\sum_{x \in S(c, r)} \min \left\{I(x), I_{A B}\right\}}{\sum_{x \in S(c, r)} 1}-\alpha \cdot \frac{\sum_{x \in R(c, r)} \min \left\{I(x), I_{A B}\right\}}{\sum_{x \in R(c, r)} 1},
$$

where $I(x)$ is the image intensity at voxel $x, I_{A B}$ denotes the average image intensity in the aortic bulbus and $\alpha$ is a weighting factor. The threshold $I_{A B}$ is used to reduce the influence of calcifications and it is computed using the model-based segmentation result. The coronary ostium position is found with a complete search on the candidate patch while varying $r$ between $2 \mathrm{~mm}$ and $4 \mathrm{~mm}$. The weighting factor $\alpha$ was chosen to be 0.5 . 


\subsection{Planes for Measurements}

For computing the measurements for procedure planning and providing supporting functionality such as automatic reformatting, a number of planes must be defined. These planes are derived from the adapted shape model and information encoded therein.

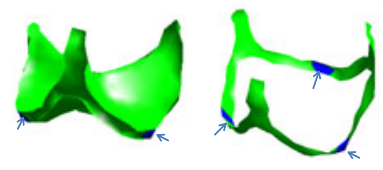

Fig. 5. Aortic valve plane landmarks, encoded on mesh

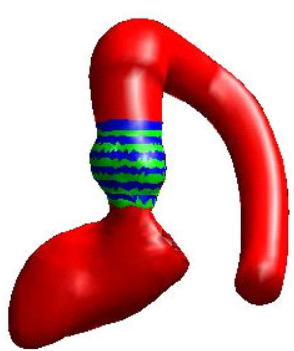

Fig. 6. Rings encoded on bulbus

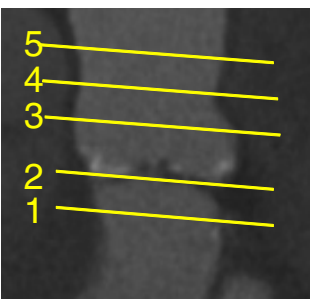

Fig. 7. Planes for diameter measurements

The aortic valve plane is defined as the plane which touches all three leaflets of the valve from beneath. To facilitate its computation, three landmarks are placed on the mesh on the basal ring of the aortic annulus (see Fig. 5). After segmentation by model adaptation, the aortic valve plane is computed from the position of the three landmarks.

To define the planes for diameter measurements of the left ventricular outflow tract (1), aortic valve annulus (2), the middle of the aortic bulbus (3), the sinutubular junction (4) and the ascending aorta (5), several rings are encoded on the mesh (see Fig. 6. 7). After model adaptation, planes are derived from respective rings by regression analysis.

\subsection{Measurements}

Diameters along aortic bulbus. The diameter information is important for selecting the proper stent [5]. The approach proposed for the measurements differs from common clinical practice, where they are currently derived from manually adjusted reformats or coronal slices of the CT image. Manual slice selection in combination with the non-circular cross-sections bears, however, a significant risk of over- or underestimating the respective quantity. The proposed 3D-based approach aims at delivering more consistent measurements.

To characterize the diameter, the mesh is cut by the respective plane. Then, an ellipse 9] or an inner and outer circle is fitted to the vertices of the resulting contour. The ventricular outflow tract and the aorta are normally elliptical, so here an ellipse is fitted using direct least square fitting (see Fig. 8, left). The aortic annulus and bulbus, however, have the shape of a rounded triangle. Here, the radius of the inner and outer circle are estimated from the vertices of the resulting contour (see Fig. 8, right). 
Distance between ostia and aortic valve plane. The distance between ostia and aortic valve plane is important to evaluate the risk of ostia occlusion. The valve leaflets are pressed against the wall of the aortic bulbus by the implanted aortic valve and it is essential to verify that they cannot occlude the coronary ostia [5. The difference of the distance of the ostia to the valve plane and the outer radius of the valve annulus can be used to asses the risk. Additionally, the distance is important for the decision, whether a specific stent can be implanted, where the upper part of the stent could occlude the coronary ostia [5].

Given the location of the ostia and the aortic valve plane, computation of the distances between the ostia and the aortic valve plane is straightforward (see Fig 9). The right image of Fig 9 also shows why it is difficult to determine the valve plane manually in $2 \mathrm{D}$ slices: As the valve is not a planar structure it touches the plane only at three points. If a slice like the one in the image is chosen to select the plane manually, the result is not correct.
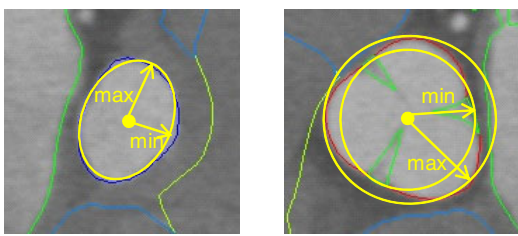

Fig. 8. Left: Fitted ellipse with short and long axis, right: fitted inner and outer circle

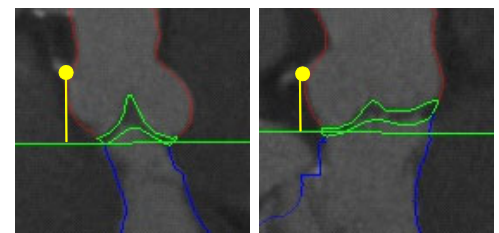

Fig. 9. Distance ostia to aortic valve plane

\section{Evaluation Setup and Results}

For the evaluation, a leaving-one-out study was conducted. $20 \mathrm{CT}$ datasets (9 with calcified valves) with reference segmentations were available for this study. Always 19 of the 20 dataset were used to train the model. This includes computation of the mean shape, boundary detectors and the ostia search regions. The model was then used to segment the remaining dataset and the measurements were conducted as described above. Then, the mean surface-to-surface error [3] of the automatic segmentation and the reference segmentation were computed and the measurements from the automatic segmentation were compared with the measurements derived from the reference segmentation.

The segmentation was successful in all 20 datasets. The mean surface-tosurface error for left ventricular outflow tract, aortic valve and aortic bulbus was $0.5 \pm 0.4 \mathrm{~mm}$. Example images of the segmentation are shown in Fig. 10. The localization of the ostia was successful for 39 out of 40 ostia (20 right, 20 left ostia). For the failure case, the landmark was detected in the coronary artery but not in the centre of the ostium. For the remaining 39 ostia, the left coronary ostium was detected with root mean squared (RMS) error of $1.2 \pm 0.6 \mathrm{~mm}$ and the right coronary ostium with an RMS error of $1.0 \pm 0.8 \mathrm{~mm}$. The valve plane was always detected successfully. The centre of the valve annulus was detected 


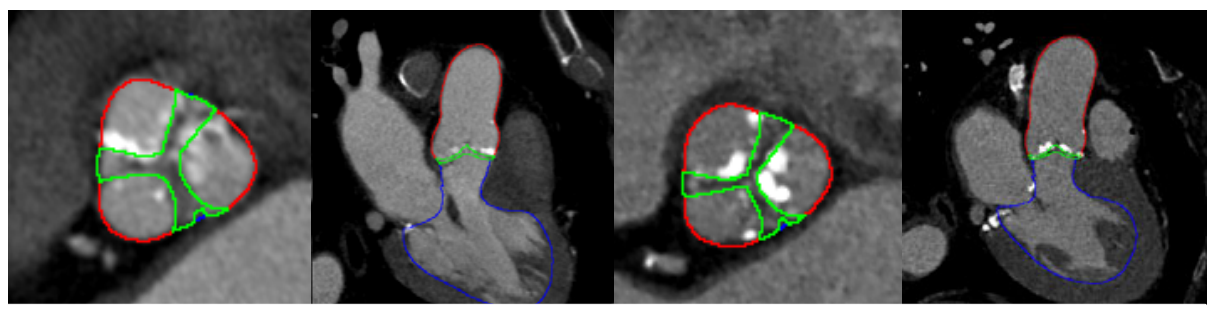

Fig. 10. Examples of aortic valve segmentation

with a RMS error of $0.8 \pm 0.3 \mathrm{~mm}$ and the normal of the plane had an RMS error of $1.8 \pm 1.0^{\circ}$.

For the different proposed measurements, the range of values (mean, stdev, min, max) was computed on the reference meshes and the RMS errors between the measurements derived from the automatically segmented data and the measurements derived from the reference meshes were computed. An overview of the measurements is given in Table 1 .

Table 1. Evaluation of measurements (all values in $\mathrm{mm}$ )

\begin{tabular}{lccccc}
\hline Measurement & mean & stdev & min & max & RMS Error \\
\hline Distance left ostium - aortic valve plane & 16.6 & 3.3 & 10.4 & 22.7 & 0.9 \\
Distance right ostium - aortic valve plane & 17.5 & 2.8 & 13.2 & 22.0 & 0.6 \\
Short axis diameter, LV outflow tract & 19.7 & 1.9 & 15.5 & 23.9 & 1.0 \\
Long axis diameter, LV outflow tract & 28.2 & 2.3 & 23.5 & 33.7 & 1.2 \\
Inner diameter, aortic valve annulus & 22.6 & 2.2 & 18.3 & 27.4 & 0.8 \\
Outer diameter, aortic valve annulus & 28.0 & 2.1 & 24.4 & 32.2 & 1.0 \\
Inner diameter, mid bulbus & 29.6 & 3.0 & 22.8 & 35.2 & 0.6 \\
Outer diameter, mid bulbus & 35.8 & 3.9 & 28.3 & 43.3 & 0.7 \\
Short axis diameter, sinutubular junction & 26.4 & 2.5 & 21.7 & 31.0 & 0.7 \\
Long axis diameter, sinutubular junction & 28.2 & 3.0 & 22.1 & 32.9 & 1.0 \\
Short axis diameter, ascending aorta & 27.1 & 2.2 & 22.3 & 31.7 & 0.5 \\
Long axis diameter, ascending aorta & 28.5 & 2.1 & 23.8 & 32.9 & 0.4 \\
\hline
\end{tabular}

\section{Conclusions}

We have presented a model-based approach for the extraction of the aortic valve geometry from CT images and showed on $20 \mathrm{CT}$ data sets that model adaptation can be done with a surface-to-surface error of $0.5 \mathrm{~mm}$. Furthermore, we presented a new approach for the detection of the coronary ostia that applies a simple pattern search method in regions encoded on the model. Accurate model adaptation in combination with the locality introduced in this way avoids misleading detection results and enables coronary ostia detection with a success rate of $97.5 \%$. We believe that this approach can be generalized and also be used for the detection of other anatomical landmarks. 
Furthermore, the resulting aortic valve model and information encoded therein was used to derive a number of measurements for planning minimally invasive aortic valve procedures. Compared to the measurements derived from the reference annotations, automatic segmentation resulted in RMS errors of the measurements between 0.4 and $1.2 \mathrm{~mm}$. Respective errors are comparable or smaller than the difference reported, for instance, for diameter measurements of the aortic valve annulus between $\mathrm{CT}$ and transthoracic echo $(1.22 \pm 1.3 \mathrm{~mm})$ or $\mathrm{CT}$ and transesophageal echo $(1.52 \pm 1.1 \mathrm{~mm})$ [10. As inaccuracies in sizing the aortic valve annulus can result in various complications such as paravalvular leaks, valve embolization or migration, centrivalvular leaks, premature leaflet deterioration or post-procedure heart block, we think that our approach has not only the potential to improve consistency of clinical measurements, but may also contribute to an improved clinical outcome.

In the future, the model may also be used to support interventional guidance. Similar as proposed for electrophysiological procedures, the aortic valve model may be overlaid onto X-ray images for that purpose. Another approach to support guidance would be the combination with methods to localize and track the aortic valve prosthesis as proposed by Karar et al. [11.

\section{References}

1. Cribier, et al.: Early experience with percutaneous transcatheter implantation of heart valve prosthesis for the treatment of end-stage inoperable patients with calcific aortic stenosis. J. Am. Coll. Cardiol. 43(4), 698-703 (2004)

2. Walther, et al.: Transapical minimally invasive aortic valve implantation: multicenter experience. Circulation 116(11 Suppl.), 1240-1245 (2007)

3. Ecabert, et al.: Automatic model-based egmentation of the heart in CT images. IEEE TMI 27(9), 1189-1201 (2008)

4. Ionasec, et al.: Dynamic model-driven quantitative and visual evaluation of the aortic valve from 4D CT. In: Metaxas, D., Axel, L., Fichtinger, G., Székely, G. (eds.) MICCAI 2008, Part I. LNCS, vol. 5241, pp. 686-694. Springer, Heidelberg (2008)

5. Tops, et al.: Noninvasive evaluation of the aortic root with multislice computed tomography implications for transcatheter aortic valve replacement. JACC Cardiovascular Imaging 1(3), 321-330 (2008)

6. Gessat, et al.: A planning system for transapical aortic valve implantation. SPIE Medical Imaging 7261, 72611E.1-72611E.12 (2009)

7. Peters, et al.: Segmentation of the heart and major vascular structures in cardiovascular CT images. SPIE Medical Imaging 6914, 691417.1-691417.12 (2008)

8. Peters, et al.: Optimizing boundary detection via simulated search with applications to multi-modal heart segmentation. Medical Image Analysis 14(1), 70-84 (2010)

9. Fitzgibbon, et al.: Direct least square fitting of ellipses. IEEE Trans. PAMI 21(5), 476-480 (1999)

10. Messika-Zeitoun, et al.: Multimodal assessment of the aortic annulus diameter: implications for transcatheter aortic valve implantation. J. Am. Coll. Cardiol. 55(3), 186-194 (2010)

11. Karar, et al.: Localization and tracking of aortic valve prosthesis in $2 \mathrm{D}$ fluoroscopic image sequences. SPIE Medical Imaging 7261, 72611Q.1-72611Q.8 (2009) 